\title{
Efficient computation of the pressures developed during high-speed train passing events
}

\author{
Lutz Hermanns $^{\mathrm{a}, *}$, J. Germán Giménez ${ }^{\mathrm{b}}$, Enrique Alarcón ${ }^{\mathrm{c}}$ \\ ${ }^{a}$ Centre for Modelling in Mechanical Engineering, University of Technology Madrid, F2I2-CEMIM, \\ cl Jose Gutierrez Abascal, 2, 28006 Madrid, Spain \\ ${ }^{\mathrm{b}}$ Contrucciones y Auxiliar de Ferrocarriles (CAF), S.A.J.M. Iturrioz, 26, 20200 Beasain, Spain \\ ${ }^{\mathrm{c}}$ Department of Structural Mechanics and Industrial Constructions, ETSII, University of Technology Madrid, \\ c/ Jose Gutierrez Abascal, 2, 28006 Madrid, Spain
}

\begin{abstract}
The paper resumes the results obtained applying various implementations of the direct boundary element method (BEM) to the solution of the Laplace Equation governing the potential flow problem during everyday service manoeuvres of high-speed trains. In particular the results of train passing events at three different speed combinations are presented. Some recommendations are given in order to reduce calculation times which as is demonstrated can be cut down to not exceed reasonable limits even when using nowadays office PCs. Thus the method is shown to be a very valuable tool for the design engineer.
\end{abstract}

(C) 2004 Elsevier Ltd. All rights reserved.

Keywords: Boundary element method; Laplace equation; Linear aerodynamic model; High-speed train; Train passing effects; Critical section

\section{Introduction}

During the last two decades the European high-speed rail network has become an interesting alternative to airplanes what belongs short to medium distances i. e. up to $500 \mathrm{~km}$. The average velocity reached on some connections in France exceeds $250 \mathrm{~km} / \mathrm{h}(155 \mathrm{mph})$ thereby achieving travel times even lower as that of the corresponding flight connection taking into account not only the flight time but also check in and out procedures.
However the increase in velocity is accompanied by aerodynamic phenomena which are only of minor importance in standard lines with running speeds up to $200 \mathrm{~km} / \mathrm{h}(124 \mathrm{mph})$. The situations where the aerodynamic forces play an important role are in the presence of strong cross winds and the passing of two trains in open air or tunnel.

Research communities all over the world dedicated a lot of time and money during the last years to the simulation of the above mentioned situations using different approaches. The TRANSAERO-Project was the first project within the Brite/Euram framework of the European Union where the companies operating high-speed lines joined their forces to investigate the aerodynamic 
effects appearing in everyday service. It found continuation in the RAPIDE project, which focussed on train passing effects. A comprehensive overview of the results obtained during the TRANSAERO project may be found in [1].

A number of different numerical tools were developed to deal with the different scenarios. Problems dominated by one dimensional wave propagation like the passing inside a tunnel have been studied extensively with implementations of the method of characteristics like the code AEROTUN developed by Prof Vardy back in the $1970 \mathrm{~s}$.

While compressibility is of major importance in the study of situations inside of tunnels, it plays a minor role during passing events at open air and may thus be neglected in the aerodynamic model. Apart from that, the forces on the power car related to viscosity are much lower than the pressure induced ones which leads to the conclusion that the corresponding effects may also be neglected when the study is limited to the power car. Note that throughout this paper the terms power car, leading car and head car are used as synonyms to make reference to the first car of a train independent of incorporating traction equipment or not. The limitation of the study to the leading car is justified by the fact that it suffers higher moments and forces than the other coaches. Adding the fact, that the design of nowadays highspeed train head cars present only few sharp edges where separation of flow is likely to occur and supposing an irrotational flow one arrives at a linear aerodynamic model. Another point to mention is that the boundary layer thickness at the leading car is very small however, at the end car of a train, which is usually $200 \mathrm{~m}$ long or even more, it may exceed $1 \mathrm{~m}$.

The linear aerodynamic model received much attention in the 1960s when Hess and Smith worked at Douglas Aircraft Company and published various papers [2-4] resuming the work carried out using this model. They used singularity distributions over quadrilateral elements, called panels, in order to calculate the velocity field. Their method became very popular in the aeronautical community due to the short calculation times needed and good results the method produced. Steinheuer [5] used the same approach in 1979 to calculate the unsteady pressures during passing manoeuvres and tunnel entries of trains. During the 1980s the progress in computer technology permitted first applications of Computational Fluid Dynamics (CFD) during the design process of high-speed trains however, the computational cost of transient simulations was still prohibitive. It was not until the 1990 s when transient simulations of $3 \mathrm{~d}$ models were carried out using CFD codes.

During the last years the engineering community commenced to deal with the coupled problem, i.e. to take into account that the aerodynamical loads, and not only these, produce displacements of the coaches thereby changing the geometry of the aerodynamic model which should be accounted for. The treatment of the coupled problem is usually performed with the linear aerodynamic model and Multi Body Simulation tools (MBS) as employed by Carrarini [6]. The commercial MBS codes include contact models for the wheel/rail contact as well as models to simulate the wheel excitation due to rail irregularities and other excitation sources thereby accounting for the most important phenomena which may contribute to a change of the aerodynamic model's geometry. The combination of the linear model and a limited number of degrees of freedom of the MBS model leads to reasonable simulation times. It is thus a valuable tool during the design process in order to identify critical suspension parameters and critical running situations.

During a train passing event the power car is submitted to high lateral forces accompanied by a yaw moment, both provoked by the high pressure zone the opposite train moves in front of its nose and the low pressure zone located behind the head of its leading car. The effect on the first car is much more pronounced than on the other cars. As the power car of the first generation high-speed trains incorporated a lot of the heavy electrical equipment, this was not of special concern, however the actual trend goes into the direction to distribute the electrical equipment as well as the driven axles along the train. The advantage of this redistribution is obvious, the passenger capacity increases however, the other side of the coin is that special care has to be taken when designing the suspension characteristics of the power cars to prevent critical situations of running safety to occur during everyday service. Even an aerodynamically proper designed high-speed train is not protected from high overturning moments produced by strong wind gusts. This lead to the implementation of wind speed forecasting systems on high-speed lines in Japan, France and Germany.

In the boundary element context an increase in efficiency is often related to implementations using multi-pole expansion or adaptive cross approximation however, as the number of unknowns one deals with in train passing scenarios usually not exceeds 10000 , even a classical implementation of the BEM does not present problems in terms of memory requirements. As will be shown the computation time may be reduced significantly by exploiting problem specific features of train passing scenarios.

This paper is concerned with the application of the direct boundary element method to the passing of two high-speed trains at open air. The paper is organised as follows. In Section 2 the problem formulation and the basic equations are described. In Section 3 the boundary element formulation and some implementation details are presented while in section 4 the results obtained for the passing of two high-speed trains 
with equal geometry are described. Section 5 contains some conclusions and proposals for future implementations.

\section{Problem formulation and basic equations}

As already mentioned in the introduction the linear aerodynamic model used in the present study has its limitations however, as the simulation is limited to the leading car it is believed that the results are in good agreement with those obtained by much more sophisticated models like the Reynolds Averaged Navier Stokes equations (RANS).

Fig. 1 presents the distribution of the pressure coefficient $C_{p}$ on the upper part of the power cars head of a single train simulation running at open air without cross wind. The results obtained with the boundary element implementation are in good agreement with the RANS solution. Perhaps the most important aspect is the calculation time employed. While the boundary element solution took about 5 min the RANS solution took several hours. It should be noted that the chosen aerodynamical model leads to reasonable results in the presence of crosswinds only if the resulting attack angle as a combination of the vehicle speed and direction and the wind component remains below a few degrees.

In the following the basic equations governing the aerodynamic phenomena are briefly described.

In an irrotational solenoidal flow of an incompressible fluid the velocity $\vec{V}$ may be derived from a scalar potential $u$ as follows:

$\vec{V}=\nabla u$
The general form of the mass conservation equation

$\frac{\partial \rho}{\partial t}+\nabla \cdot(\rho \vec{V})=0$

with density $\rho$ reduces for an incompressible fluid to the following form:

$\operatorname{div} \vec{V}=0$

Substituting $\vec{V}$ in (3) by (1) results in the well known Laplace Eq. (4).

$\operatorname{div} \nabla u=\Delta u=0$

Note that the Laplace equation is independent of time, the unsteady pressure fluctuations during the passing of the trains will be introduced by means of the time derivative of the potential $u$ in the Bernoulli Equation (5).

$$
\begin{aligned}
p= & p_{\infty}+\rho\left\{\vec{V}_{0} \cdot \nabla u-\frac{1}{2}\left[\left(\frac{\partial u}{\partial x}\right)^{2}+\left(\frac{\partial u}{\partial y}\right)^{2}\right.\right. \\
& \left.\left.+\left(\frac{\partial u}{\partial z}\right)^{2}\right]-\frac{\partial u}{\partial t}\right\}
\end{aligned}
$$

where $p_{\infty}$ denotes the reference pressure and $\vec{V}_{0}$ describes the stationary translational motion of the moving frame of reference fixed to the object the pressure is calculated on. The time derivative of the potential may be approximated by a finite difference scheme.

The boundary conditions for the flow have to guarantee that the normal component of the relative velocity on the objects walls vanish, i.e. that the normal component of the absolute velocity is equal to the velocity of the object's wall. This is accomplished by prescribing the normal velocity as follows:

$\nabla u \cdot \vec{n}=\vec{V}_{0} . \vec{n}$

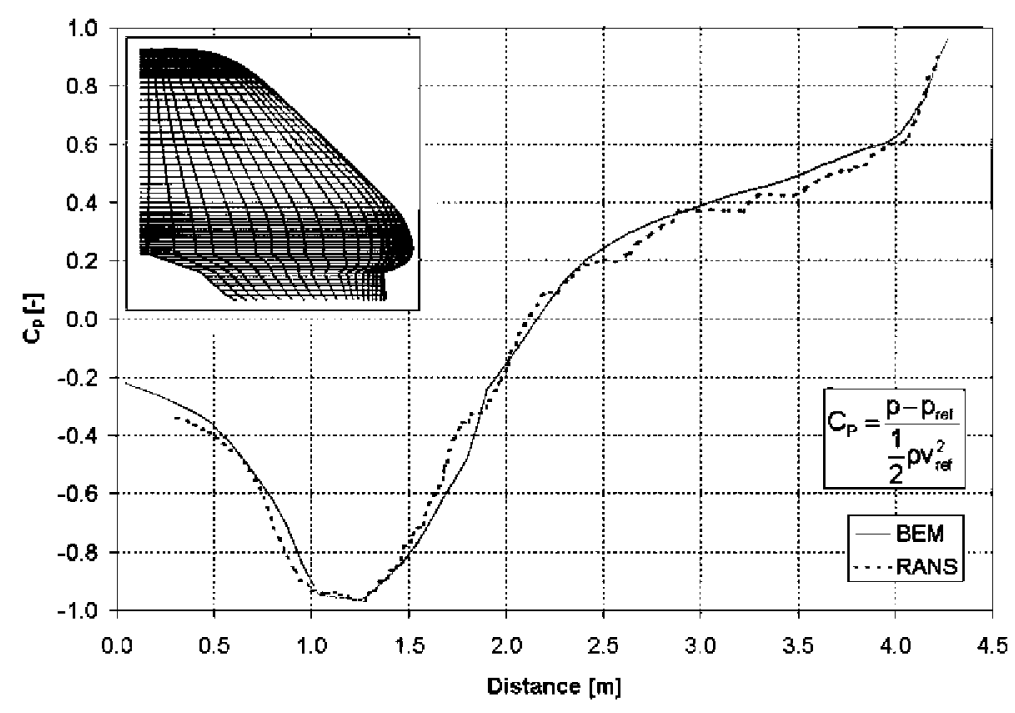

Fig. 1. $C_{p}$ on the upper part of a train nose. 
where $\vec{n}$ denotes the normal vector of the surface element pointing from the fluid region into the object.

\section{Boundary element formulation}

For a detailed derivation of the direct boundary element formulation in the framework of weighted residual methods the reader is referred to standard textbooks on boundary element methods like [7], [8], or [9]. For completeness in the following the required steps to arrive at the boundary element formulation, in particular at an implementation of the collocation method, are briefly described.

Considering a potential function $u$ on a domain $\Omega$ which is required to satisfy Eq. (4) inside $\Omega$ and the following two sets of boundary conditions

$u=\bar{u}$ on part $\Gamma_{1}$ of the boundary of $\Omega$ $\partial u / \partial n=\bar{q}$ on part $\Gamma_{2}$ of the boundary of $\Omega$

where the total boundary of $\Omega$ is defined as $\Gamma=\Gamma_{1}+\Gamma_{2}$, a weighting function $u^{*}$, which represents the fundamental solution of the field equation, with continuous first derivatives can be introduced to arrive at the following integral equation:

$$
\begin{aligned}
u^{i} & +\int_{\Gamma_{2}} u q^{*} \mathrm{~d} \Gamma+\int_{\Gamma_{1}} \bar{u} q^{*} \mathrm{~d} \Gamma \\
& =\int_{\Gamma_{2}} \bar{q} u^{*} \mathrm{~d} \Gamma+\int_{\Gamma_{1}} q u^{*} \mathrm{~d} \Gamma
\end{aligned}
$$

where $q=\partial u / \partial n$ and $q^{*}=\partial u^{*} / \partial n$. Note that Eq. (7) is valid for any point inside the domain $\Omega$, but to arrive at a boundary formulation it has to be taken to the boundary. This step includes a splitting of integrals and limiting processes which are nontrivial, therefore the reader is once more referred to the textbooks mentioned above for a rigorous derivation. Here we will only present the result, which is valid for a point on the boundary.

$$
\begin{aligned}
c^{i} u^{i} & +\int_{\Gamma_{2}} u q^{*} \mathrm{~d} \Gamma+\int_{\Gamma_{1}} \bar{u} q^{*} \mathrm{~d} \Gamma \\
= & \int_{\Gamma_{2}} \bar{q} u^{*} \mathrm{~d} \Gamma+\int_{\Gamma_{1}} q u^{*} \mathrm{~d} \Gamma
\end{aligned}
$$

The free term coefficient $c^{i}$ depends on the local geometry of the point under consideration. While Eq. (8) describes an integral equation for interior problems, the train passing case is an exterior Neumann problem. That means that the integrals over $\Gamma_{1}$ can be dropped as they do not really exist in this particular problem. In the derivation of Eq. (8) the second Green's Identity has been applied which is valid only for closed regions. In order to treat exterior problems one has to close the model artificially by means of a sphere whose radius $R$ tends to infinity. This leads to the following equation.

$$
\begin{aligned}
c^{i} u^{i} & +\int_{\Gamma_{2}} u q^{*} \mathrm{~d} \Gamma+\int_{\Gamma_{\text {sphere }}} u q^{*} \mathrm{~d} \Gamma \\
= & \int_{\Gamma_{\text {sphbere }}} q u^{*} \mathrm{~d} \Gamma+\int_{\Gamma_{2}} \bar{q} u^{*} \mathrm{~d} \Gamma
\end{aligned}
$$

It can be shown that the following relation holds for any problem satisfying regularity conditions as $R$ tends to infinity [7].

$\lim _{R \rightarrow \infty}\left[\int_{\Gamma_{\text {sphere }}} u q^{*} \mathrm{~d} \Gamma-\int_{\Gamma_{\text {sphere }}} q u^{*} \mathrm{~d} \Gamma\right]=0$,

leading to the following boundary integral equation for exterior Neumann problems:

$c^{i} u^{i}+\int_{\Gamma} u q^{*} \mathrm{~d} \Gamma=\int_{\Gamma} \bar{q} u^{*} \mathrm{~d} \Gamma$,

where the identity $\Gamma=\Gamma_{2}$ has been used. When calculating the integrals the normal vectors point from the fluid region into the cavity i.e. the train.

Eq. (11) is the starting point for a numerical implementation of the boundary element method for exterior Neumann problems.

An implementation of the boundary element method starts by dividing the boundary $\Gamma$ of the domain $\Omega$ into $m$ elements which, in the approach used, share not only the geometrical nodes but also the support nodes for the field variable interpolation, i.e. an isoparametric continuous approach with quadrilateral 4 node elements has been implemented. The variation of the element's geome$\operatorname{try} \vec{x}$ and the two field variables, potential $u$ and its normal derivative $q$, over an element $e$ may thus be described by means of a product of shape functions $N_{i}$ and the nodal values of the co-ordinates and field variables respectively:

$\vec{x}_{e}=\sum_{i=1}^{4} N_{i} \vec{x}_{e}^{i}$

$u_{e}=\sum_{i=1}^{4} N_{i} u_{e}^{i}$

$q_{e}=\sum_{i=1}^{4} N_{i} q_{e}^{i}$

where $\vec{x}_{e}^{i}, u_{e}^{i}, q_{e}^{i}$ denote the co-ordinates, the potential and its normal derivative at node $i$ of element $e$. The integrals in Eq. (11) can thus be substituted by a summation over all elements, which leads to the following statement:

$c^{i} u^{i}+\sum_{e=1}^{m} \int_{\Gamma_{e}} u_{e} q^{*} \mathrm{~d} \Gamma=\sum_{e=1}^{m} \int_{\Gamma_{e}} q_{e} u^{*} \mathrm{~d} \Gamma$

Note that the nodal values of the potential and its normal derivative may be extracted from the integrals and accumulated in vector form to arrive at a linear system of equations equal of size to the number of nodes. The resulting matrix equation may be written as follows: 
$H \vec{u}=G \vec{q}=\vec{f}$

where $H$ contains the contributions from the integrals including $q^{*}$ and the free terms while $G$ is built by the integrals containing $u^{*}$. Note that for the application treated in the present study all components of the $\vec{q}$ vector are known. After solving the linear system of equations the potentials $u^{i}$ at all nodes are known. Two directional derivatives of the potential may then be calculated, by means of the shape function derivatives and the nodal potential values, to complete a set of three velocity vectors on a surface point, the normal component, as boundary condition imposed, and two tangential ones. This set is then transformed to the global Cartesian system.

The evaluation of the diagonal coefficients of the $H$ matrix is realised by the indirect method [8], i.e. imposing a unit potential and solving the system for the diagonal coefficients as in this case the solution is known. As the problem under consideration is an exterior one the model has to be closed artificially by means of a sphere whose radius $R$ tends to infinity leading once more to Eq. (9). It can be shown that the contribution of the integral containing $q^{*}$, known as azimuthal integral, is -1 [8].

The calculation of the coefficient matrices $H$ and $G$ requires an adaptive integration scheme in order to preserve the same accuracy level over all coefficients. This is of major importance as the indirect method is used to evaluate the diagonal coefficients of the $H$ matrix. The determination of the number of Gauss Points necessary to limit the relative error to a certain value, in this case $10^{-4}$ was chosen, is performed based on the ratio of the shortest distance between collocation point and the element under consideration and the element length as proposed in [9]. Plane symmetry is exploited in order to simulate the ground effect. The applications have been coded in Fortran90. The part of the codes where the coefficient matrices are calculated is prepared to use a second CPU if present. A critical section implementation has been chosen, as the co-ordination of the threads is quite simple and speed is critical to the application. Usually the meshes of the two trains are quite similar what the number of elements belongs, therefore in the multithread versions each of the threads is used to calculate the coefficients corresponding to the unknowns of one train. As a result the threads never conflict when addressing the memory where the coefficient matrix is stored. The contributions to the rhs vector are stored separately for each thread.

Making use of the fact that the coefficients relating collocation points and elements of the same object do not change during the simulation one can reduce the calculation time considerably as will be shown.

With regard to the simulation of several speed combinations it is important to note that, as the governing differential equation is linear, the principle of superposition may be used to build new solutions as linear combinations of existing ones. That means that actually only two scenarios has to be simulated while the solution to any other scenario may be built as a linear combination of these two. If the relative displacement between the trains coincides in both scenarios, the coefficient matrix needs to be calculated only once. In combination with the treatment of two rhs vectors this leads to further reduction in computation time.

In order to evaluate the efficiency of the options described two versions of the program were coded, one of them deals with a single rhs vector while the other works with two. In Table 1 the CPU times for the integration as well as the total computation times needed for the simulation of one geometrical configuration with two sets of boundary conditions, i.e. two rhs vectors, are presented. The total computation time includes the integration of the coefficients, the solution of the system of equations, postprocessing and storing of the results. The computer employed is a HP xw 8000 workstation with two Xeon processors running at $2.66 \mathrm{GHz}$. The model used for this test case is quite large, as it comprises 11264 degrees of freedom. The memory requirements do not exceed $510 \mathrm{MB}$ as the coefficient matrix is stored in single precision variables after being calculated using double precision arithmetic. The resolution of the system of equations is performed by a GMRES(50) implementation with Modified Gram Schmidt orthogonalization. Usually about 60 iterations are needed to reduce the $L_{2}$ norm of the residuum normalised by the $L_{2}$ norm of the rhs vector below $10^{-7}$.

The reuse of the constant part of the coefficient matrix in conjunction with the treatment of two rhs vectors reduces the CPU time needed for the construction of the coefficient matrix from 1920 to 498 or $475 \mathrm{~s}$ which corresponds to $26 \%$ and $25 \%$ respectively. As the two integration threads in the multi thread version operate almost independently the resulting level of performance is very good. The total computation time achieved even with the single thread version permits to perform a complete train passing simulation, which usually comprises 150 time steps, during one day.

Table 1

Calculation times needed for the solution of one geometrical configuration with two sets of boundary conditions

\begin{tabular}{lllll}
\hline $\begin{array}{l}\text { Thread } \\
\text { model }\end{array}$ & $\begin{array}{l}\text { Reuse of } \\
\text { constant } \mathrm{H} \\
\text { matrix entries }\end{array}$ & $\begin{array}{l}\text { Two rhs } \\
\text { vectors }\end{array}$ & $\begin{array}{l}\text { CPU time } \\
\text { int. (s) }\end{array}$ & $\begin{array}{l}\text { Total comp. } \\
\text { time (s) }\end{array}$ \\
\hline Single & No & No & 1920 & 2046 \\
Single & Yes & No & 996 & 1122 \\
Single & Yes & Yes & 475 & 562 \\
Multi & No & Yes & 962 & 557 \\
Multi & Yes & Yes & 498 & 324 \\
\hline
\end{tabular}


Note that the simulation tasks can be distributed among several computers as the different times steps represent stationary solutions which are combined at the end to get the time histories of pressure, forces and moments. This type of distribution among two computers permits to carry through a complete simulation overnight. The time needed to perform a pass by simulation with the multi thread version is approximately $13.5 \mathrm{~h}$.

In the cases where the two trains have equal geometry the calculation times can be reduced even more when using identical meshes for the two trains and taking into account the point symmetry in a vertical plane however, this has not yet been implemented. An additional reduction in calculation time could be achieved by identifying the elements where the normal flow as boundary condition imposed is zero because in these cases the corresponding coefficients of the $G$ matrix don't have to be calculated as their contributions to the rhs vector vanish.

\section{Results for the train passing scenario}

The computer programs described in the previous section have been used to simulate the train passing of two high-speed trains of equal geometry at open air without cross wind.

Throughout this paper the forces and moments refer to a global Cartesian co-ordinate system where the $x$ axis points in the travel direction and $z$ is used to define the vertical direction.

Fig. 2 shows a perspective view of the mesh used for the simulation. The distance between track centres was chosen to $4.5 \mathrm{~m}$, a typical value for high-speed lines in Europe. Three speed combinations will be considered

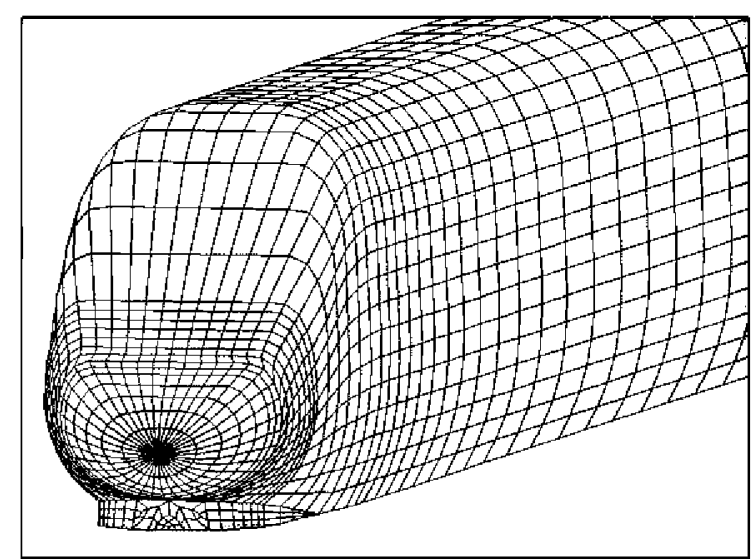

Fig. 2. Surface mesh employed for the train passing simulation.

which are resumed in Table 2. The distance between the train noses at the beginning of the simulation is about $7 \mathrm{~m}$.

During the simulation of a train passing a huge amount of results is obtained, here only a few curves, which are thought to be the most interesting ones, will be presented. Three evaluation points on a sidewall of

Table 2

Speed combinations considered

\begin{tabular}{lll}
\hline Scenario & $\begin{array}{l}\text { Velocity train A } \\
\text { in } \mathrm{km} / \mathrm{h}(\mathrm{mph})\end{array}$ & $\begin{array}{l}\text { Velocity train B } \\
\text { in } \mathrm{km} / \mathrm{h}(\mathrm{mph})\end{array}$ \\
\hline 1 & $275(171)$ & $0(0)$ \\
2 & $275(171)$ & $250(155)$ \\
3 & $275(171)$ & $275(171)$ \\
\hline
\end{tabular}

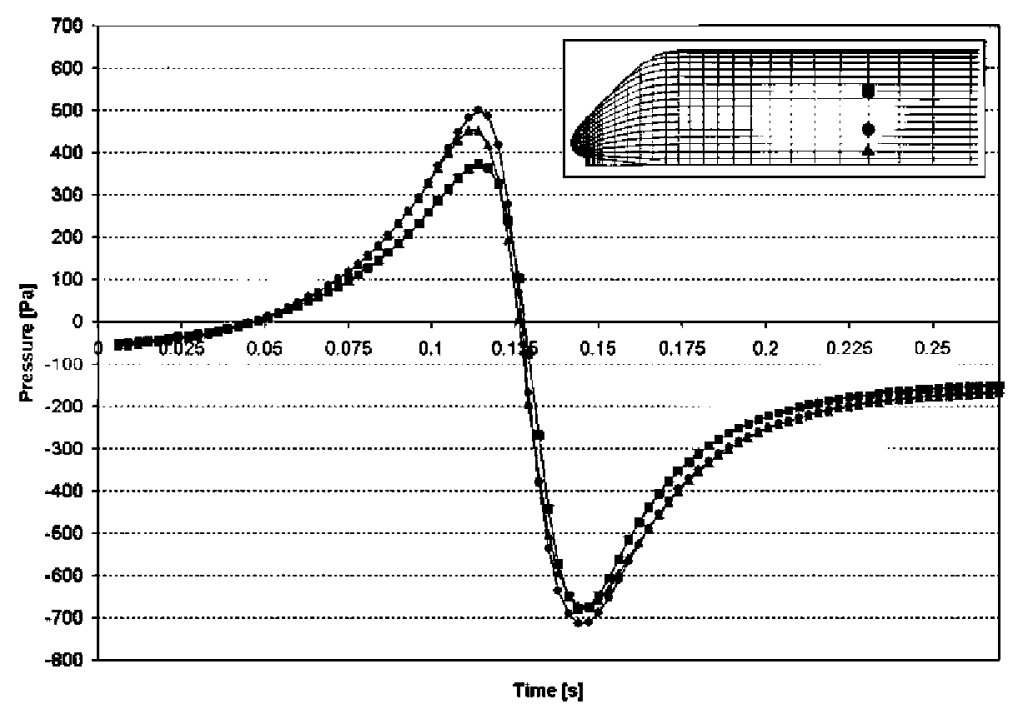

Fig. 3. Pressure time histories of train B; scenario 2. 
the trains, indicated by a square, a circle and a triangle, were selected to reflect the general tendencies observed regarding the pressure time histories.

In what follows the pressure time histories and its derivatives with respect to time of scenario 2 as well as some comparisons between the different scenarios will be presented.

Note that at the beginning of the simulation the presence of train $\mathrm{A}$ affects the pressure values at the evaluation points as a small slope is observed in Fig. 3. The extreme values are located a little above the nose of the passing train. Fig. 4 resumes the pressure time history of train A, note that the maximum and minimum pressure values are observed on the slower moving train.

The three curves presented in Fig. 5 correspond to the pressure time histories at the evaluation point marked by a filled circle of train B for all scenarios. An adimensional time defined as time divided by $10 \mathrm{~m}$ and multiplied by the average velocity of the two trains were applied. Note that not the absolute pressure is presented but the difference between the absolute and the initial one corresponding to the evaluation point, i.e.

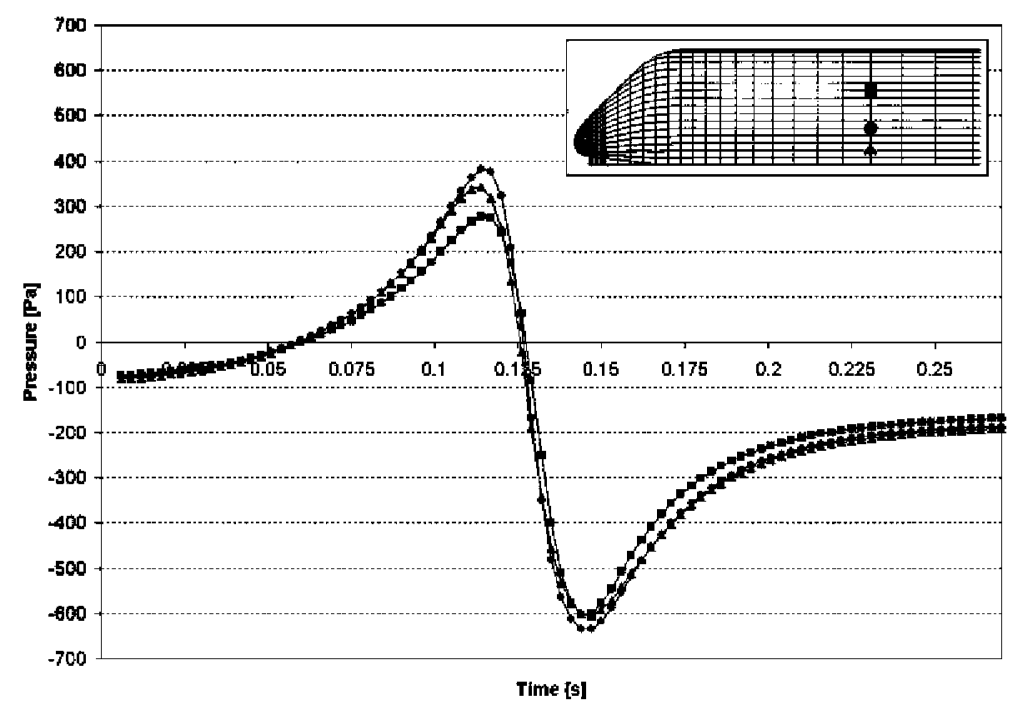

Fig. 4. Pressure time histories of train A; scenario 2.

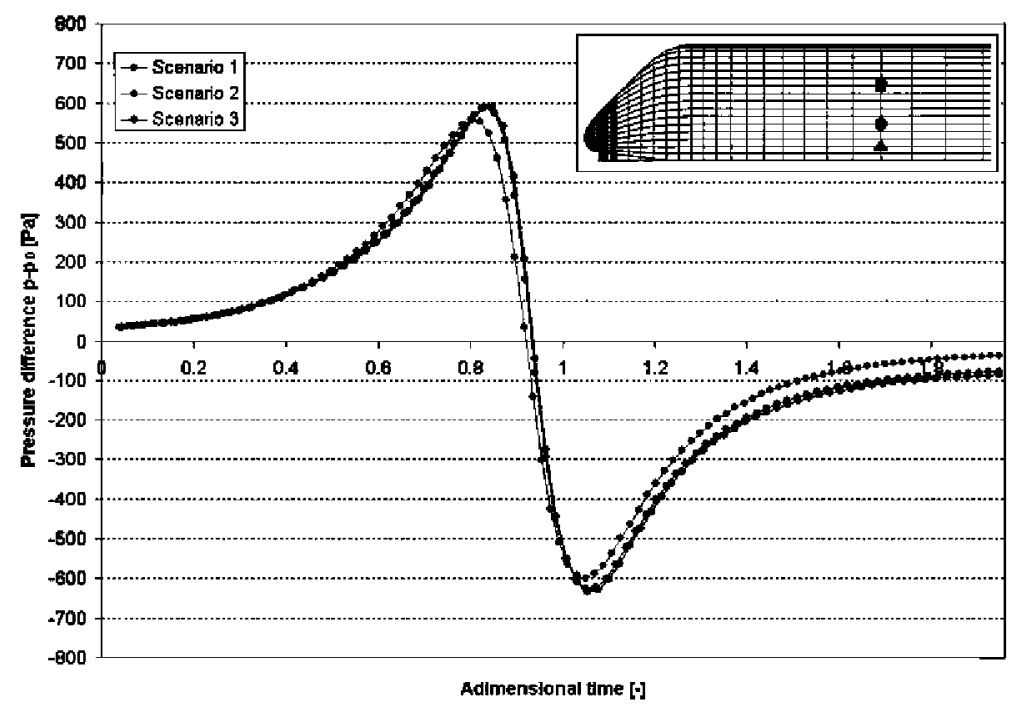

Fig. 5. Time histories of pressure differences on train B 
when the presence of the passing train does not yet affect the value. From an engineering point of view the curves of all scenarios are very similar, meaning that the extreme value of pressure difference suffered when the passing train circulates at $275 \mathrm{~km} / \mathrm{h}$ is almost identical although the running speed of the other train varies between the different scenarios.

Apart from the pressure time histories its time derivative is also of interest especially when passenger comfort is of concern. In Fig. 6 the time histories of the pressure rate for scenario 3 are presented. Nowadays high-speed trains are usually sealed to prevent the rapid pressure changes occurring at the exterior from transmitting to the interior of the coaches however, the sensors which form part of the air condition controlling system have to be positioned adequately to transmit reliable data. Based on this information the air conducts will be closed or not, thus the histories of pressure rate may help to identify proper sensor locations.

The obtained pressure values of the power car have been integrated resulting in forces and moments acting in the centre between the bogies.

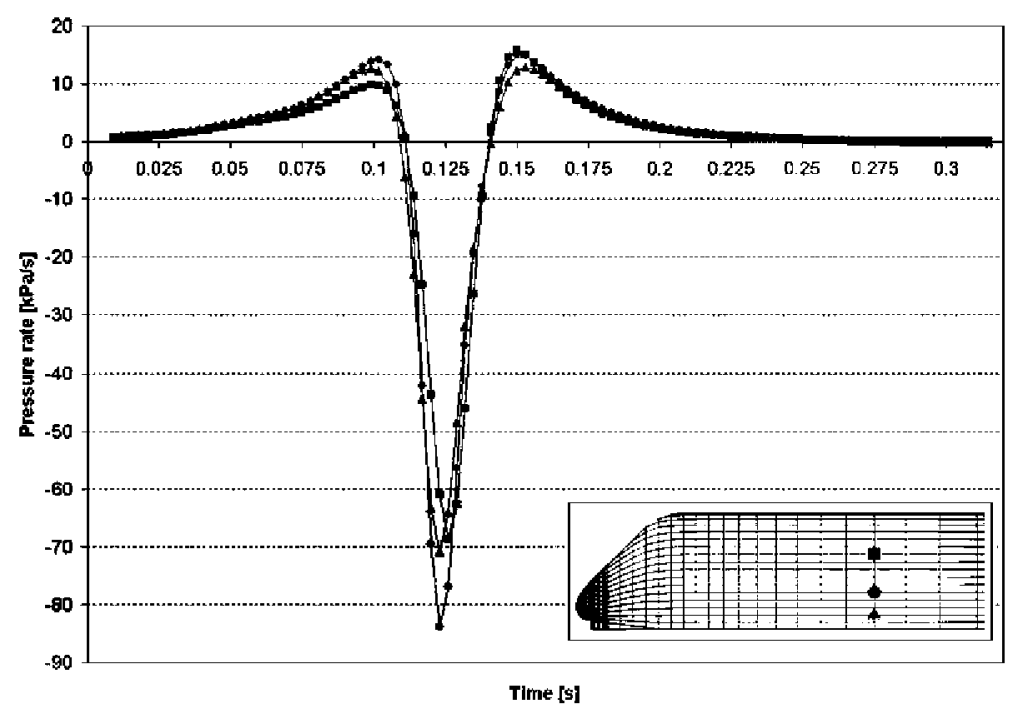

Fig. 6. Pressure rate; scenario 3.

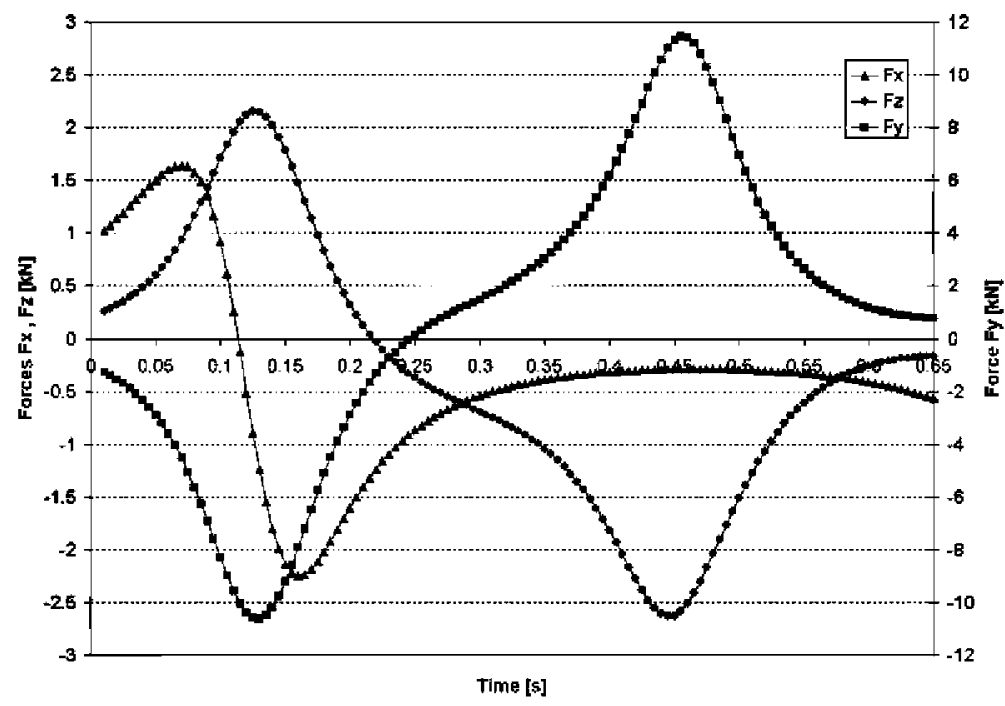

Fig. 7. Forces on train B; scenario 1 . 
While the peak pressure values on the slower moving train do not vary too much between the scenarios considered, the forces and moments do.

Fig. 7 shows the time history of the forces train $B$ is subjected to during the passing of train A for scenario 1. The most important one is $F_{y}$ as it contributes to the overturning moment $M_{x}$ as well as to the yaw moment $M_{z}$ which may lead to important horizontal displacements in the secondary suspension when considered simultaneously with forces derived from cross wind or alignment failures.
In Fig. 8 the forces corresponding to scenario 2 are shown. Note that in this case the train moves into the opposite direction as can easily be verified by the sign of $F_{x}$ at the beginning of the simulation. The lateral force $F_{y}$ increased by $57 \%$ with respect to scenario 1 . The moments corresponding to both scenarios show the same tendency though much more pronounced, i.e. yaw moment $M_{z}$ of scenario 2 is $98 \%$ higher than the corresponding moment of scenario 1 . The time histories of the moments are shown in Figs. 9 and 10 for both scenarios.

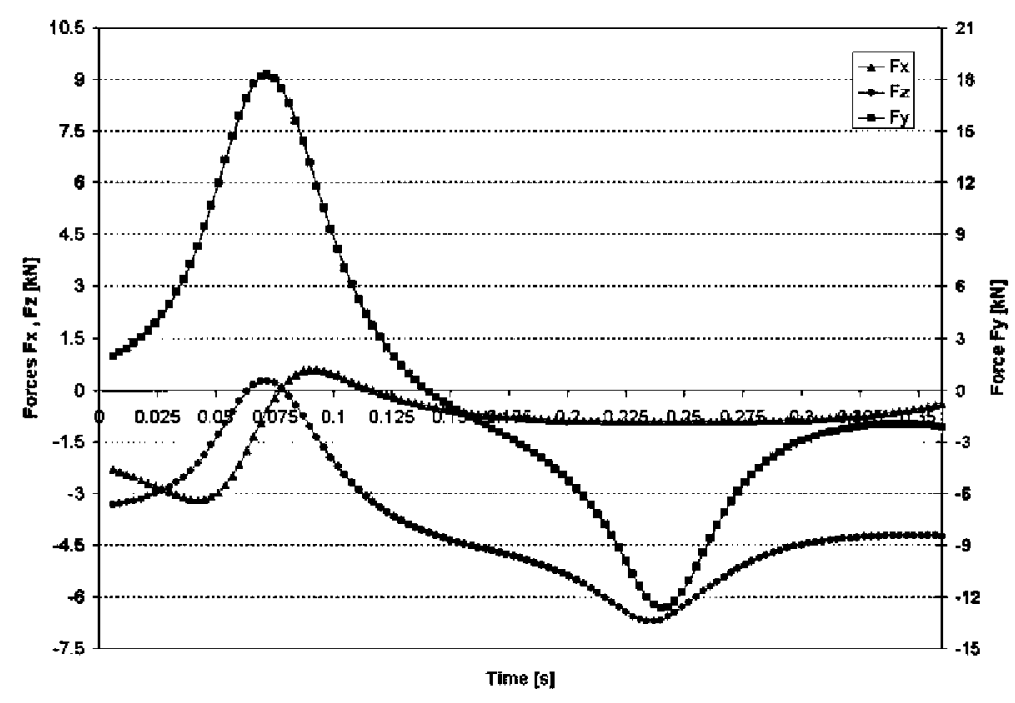

Fig. 8. Forces on train B; scenario 2.

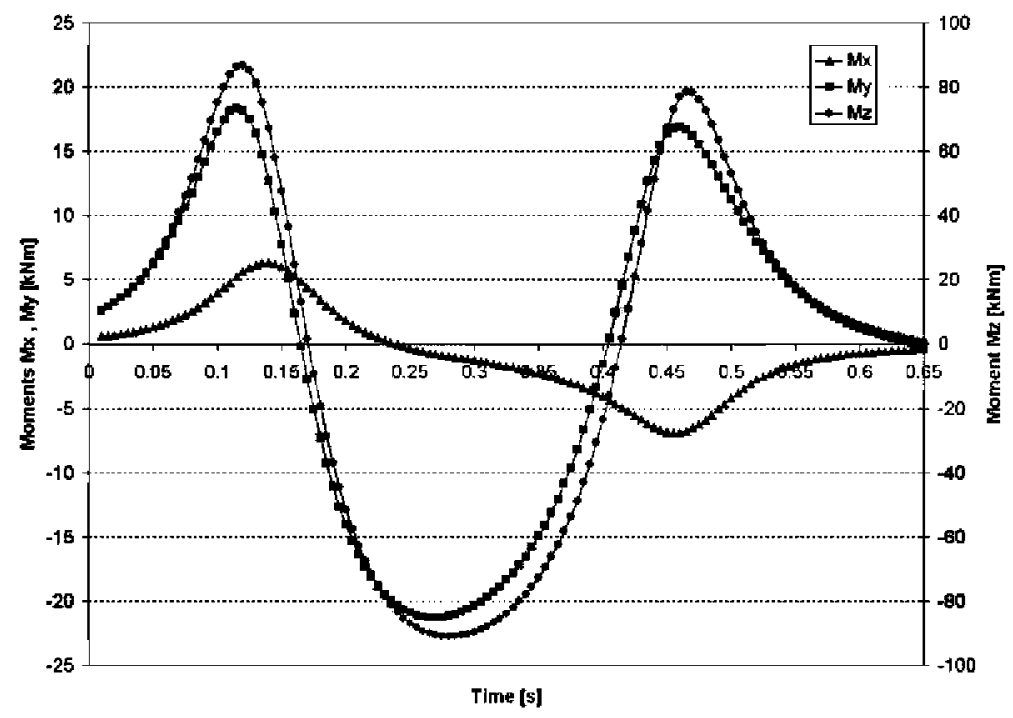

Fig. 9. Moments on train B; scenario 1. 


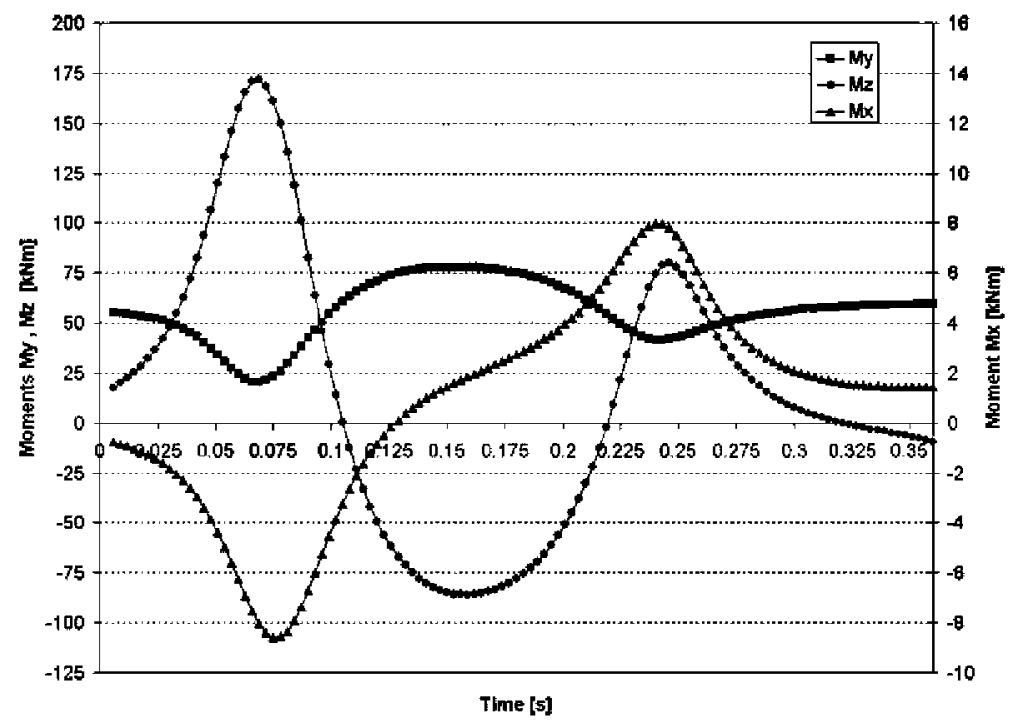

Fig. 10. Moments on train B; scenario 2.

The forces and moments for scenario 3 are only a few percent higher than for scenario 2 therefore the curves will not be presented here.

Before applying the forces and moments to finite element or MBS models the aerodynamical simulation should be extended in time into both directions in order to catch the rise at the beginning and the constant values at the end of the passing scenario. Note that with end of passing scenario not the passing of the whole trains is meant but the time when forces and moments on the power car reach constant values. For the MBS or finite element simulation this constant values should be applied throughout the rest of the simulation. This type of uncoupled simulation between aerodynamical and mechanical model does obviously not account for the displacements of the train during the simulation, i.e. the results of the mechanical system does not affect the aerodynamical one. As mentioned in [10] where both uncoupled and coupled models were used to simulate a train passing scenario, the differences between the results obtained used to be negligible. As the distance between track centres in the present study is quite high it is believed that an uncoupled simulation would be the method of choice as the coupling effect will be very small.

\section{Conclusions}

The present paper resumes the results obtained applying various implementations of the direct boundary element method to solve the Laplace equation, which governs the flow field around the power car of high-speed trains in an approximative manner. It is dem- onstrated that the BEM produces results which are in good agreement with those obtained by much more sophisticated models like RANS. In addition to the advantage of the BEM over domain type methods like finite volume, or finite element formulations with respect to meshing issues, the reduced calculation time needed permits to simulate the entire passing of the two power cars in a reasonable amount of time on nowadays office PCs. It should be mentioned that these calculation times are achieved although no concessions are made with respect to the accuracy of the coefficient matrices as an adaptive integration scheme has been implemented.

The fact that the Laplace equation describes a stationary behaviour permits to calculate the "exact" approximate solution at any time instant without knowledge of the previous solution history. In addition, an important part of the coefficient matrices does not change with time because the relative distances between collocation points and elements of the same moving object are constant. It is thought that thanks to these two points model reduction techniques using the Proper Orthogonal Decomposition may drastically reduce the calculation times when considering other distances between the tracks like $4.3 \mathrm{~m}$ or $4.7 \mathrm{~m}$. An implementation for $2 \mathrm{~d}$ problems has given very promising results.

The yaw and overturning moments resulting from a train passing event may seriously affect the train's running safety. It is clear from the results obtained that the slower moving train is subjected to higher moments considering two trains with identical design. By using adimensional coefficients for the forces and moments in conjunction with an adimensional time one can investigate additional speed combinations being the ratio of 
the velocities the same. Although the worst case which consists in being passed by a future high-speed train running at a velocity substantially higher than the ones considered in the present study is not covered, they can be easily investigated using the coefficients mentioned above. In general there exists a tendency towards smoother designs what belongs the nose of the power car of new generation of high-speed trains which may alleviate the effects caused by the increase in velocity.

The resulting forces and moments the power car is subjected to can be applied to a mechanical model in order to study the influence of the aerodynamical loads combined with those induced due to rail irregularities or alignment failures on the running safety of the train. This type of uncoupled simulation is thought to be sufficient in terms of accuracy as the lateral displacements of the car bodies usually not exceed a few centimetres while the free space between the car bodies of two passing trains is usually around $1.5 \mathrm{~m}$ considering a distance between tracks of $4.5 \mathrm{~m}$.

\section{Acknowledgments}

The contribution of CONSTRUCCIONES Y AUXILIAR DE FERROCARRILES, S.A. (www.caf.es) providing the geometry data of two high-speed train prototypes as well as the permission to publicise the results presented in the present paper is gratefully acknowledged.

\section{References}

[1] Schulte-Werning, Burkhard, editors. Notes on Numerical fluid mechanics and multidisciplinary design, vol. 79. Springer; 2002, ISBN 3-540-43316-3.

[2] Hess JL. Calculation of potential flow about bodies of revolution having axes perpendicular to the free-stream direction. J Aerospace Sci 1962;29.

[3] Hess JL, Smith AMO. Calcultaion of nonlifting potential flow about arbitrary three-dimensional bodies. J Ship Res $1964 ; 8(2)$.

[4] Hess JL, Smith AMO. Calculation of potential flow about arbitrary bodies. In: Progress in aeronautical sciences, vol. 8, Pergamon Press, 1985.

[5] Steinheuer J. Calculation of unsteady pressures during passing and tunnel entrance of trains. In: Morel T, Dalton C, editors. Aerodynamics of transportation. ASME; 1979.

[6] Carrarini A. Coupled multibody-aerodynamic simulation of high-speed trains manoeuvres. Proc. of virtual nonlinear multibody systems, 2002, ISBN 1-4020-1339-6.

[7] Cañas J, París F. Boundary element method, fundamentals and applications. Oxford Press; 1997, ISBN 0-19-8565372.

[8] Brebbia C, Dominguez J. Boundary elements an introductory course. Mc Graw Hill; 1989, ISBN 0-905451-76-7.

[9] Beer G. Programming the boundary element method: an introduction for engineers. John Wiley; 2001, ISBN 0-47185722-X.

[10] Arnold M, Carrarini A, Heckmann A, Hippmann G. Modular dynamical simulation of mechatronic and coupled systems. In: Fifth World Congr. Comput. Mech., Vienna, 2002. 